\title{
Temporal Expression MicroRNA-21 in Serum of Patients with Spinal Cord Injury \\ Hong-yan $\mathrm{LI}^{1}$, Dong-xu ZHAO ${ }^{1,{ }^{*}}$ and Ming-lei ZHANG ${ }^{1}$ \\ ${ }^{1}$ China-Japan Union Hospital of Jilin University, 126 Xian-Tai Street, Chang Chun 130033, China. \\ ${ }^{*}$ Corresponding author
}

Keywords: Spinal cord injury, Serum, MicroRNA, MicroRNA-21

\begin{abstract}
Objective: To investigate the levels of microRNA-21 (miR-21) in the serum of patients with spinal cord injury (SCI) and to determine whether there was a correlation with degree of injury. Methods: Quantitative real-time reverse transcription-polymerase chain reaction( q-RT-PCR)was used to measure the serum levels ofmiR-21 at 1, 7 and 28 days and 3 months after SCI 42 in male and female SCI patients $(n=48)$ and compare with age and sex-matched patients with non spinal cord injuries (NSCI, $\mathrm{n}=60)$ and healthy volunteers $(\mathrm{n}=70)$. Correlations between serum miR-21 levels and age, sex and degree of injury were investigated. Results: The data from the present study show that the serum level of miR-21 immediately increased and peaked on day 7 post-SCI and then declined to the control level. There were no differences between NSCI group and normal control group at each time point. The expression level o fmiR-21 was related to the lesion degree of SCI. The age and sex of the patients did not influence miR-21 expression after SCI. Conclusions: These finding might provide reference for diagnosis and treatment and contribute to the identification of selective and temporal drug targeted therapy after SCI.
\end{abstract}

\section{Introduction}

Traumatic spinal cord injury (SCI), one of the most leading causes of disability and mortality is one the most common and devastating injuries observed in spine and neurosurgery departments. It is usually caused by motor vehicle accidents, sports injuries, diving accidents, and violence, which can cause permanent disabilities such as paralysis and loss of movement or sensation. In addition, SCI initiates a variety of inflammatory and/or immune responses including the infiltration of leukocytes such as monocytes, macrophages, T-cells and NK cells, into the injured area, 1 which are the key factors of the secondary damage occurring subsequent to SCI.1 The treatment of SCI remains one of the greatest challenges for the basic science and clinical investigators so far. Despite many therapies have been explored, all method demonstrated some limited efficacy thus far.2 The celluar mechanisms associated with SCI are complex and involve a multitude of signaling pathways and molecular dysfunctions.3,4 MicroRNAs (miRNAs) are a novel class of small noncoding RNAs that negatively regulate gene expression at the posttranscriptional level by binding to the 3'-untranslated region of target mRNAs leading to their translational inhibition or sometimes degradation.5 It was recently reported that miRNAs are estimated to regulate $60 \%$ of all genes in the human genome. 6 thus, they may widely influence the signaling networks leading to pathological responses after SCI. Increasing reports shows that a large number of miRNAs are expressed in the central nervous system (CNS).7,8 Some miRNAs are involved in several neurological disorders, such as including traumatic CNS injuries and neurodegenerative diseases.9-15 Therefore, miRs could become attractive novel therapeutic targets for the treatment of SCI. 
MicroRNA-21 (miR-21) is an important member of the miRNA family. Recently, some studies have showed that miR-21 was increased following several types of CNS injuries, such as traumatic brain injury and brain ischemia.1 3-16 Additionally, some studies have shown the involvement of miRs in the pathogenesis of SCI in a rat contusion SCI model,10-12 with miR-21 emerging as one of the most dysregulated miRs.17 However, the expression level of miR-21 on patients with SCI are still unknown. Hence, the present study is aimed to determine the serum miR-21 in SCI patients at different time points after SCI and analyze the differential expression pattern of the miR-21 based on the time after injury, age and sex between SCI patients and age- and sex-matched non-SCI (NSCI) patients and controls. The outcome should help designing time dependent specific drug targeted therapy to SCI.

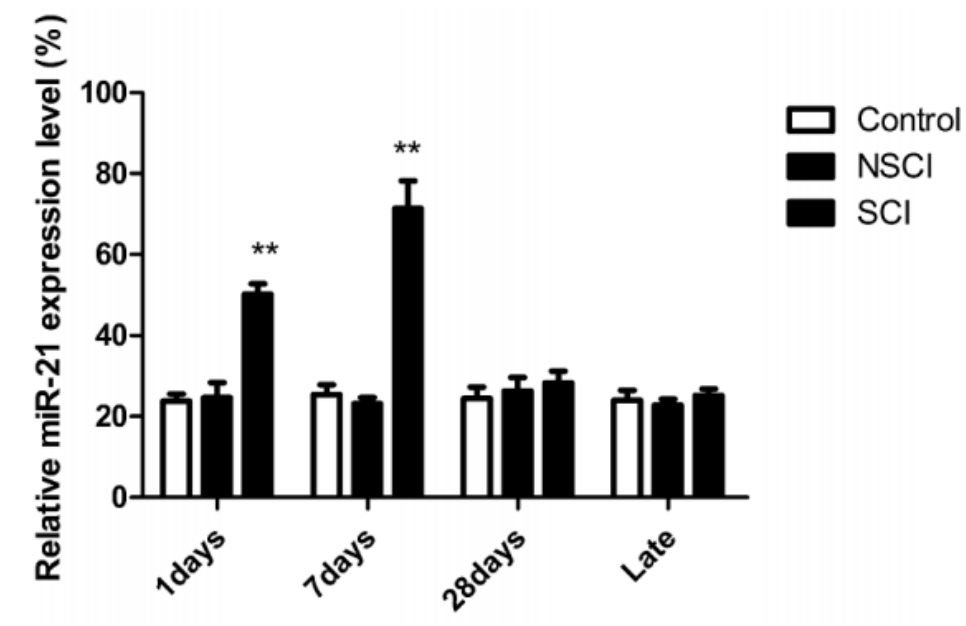

Figure1. Re lative level of micro RNA-21 (miR-21) in the serum after spinal cord injury (SCI) $(\mathrm{n}=90)$ and non-spinal cord injuries (NSCI) $(n=70)$. Serum from SCI patients and control individual (NSCI and healthy volunteers) were collected 1, 7, 28 days and at three months ( $>30$ days) after a sustained SCI (late). Data are represented as mean \pm SEM (two-way A NOVA test and Tukey HSD post hoc analys is).

$* \mathrm{P}<0.05, * * \mathrm{P}<0.01$ for SCI patients Vs control.

\section{Material and Method}

Subjects All the studies with SCI patients $(n=90)$ and NSCI patients $(n=60)$ and healthy volunteers $(n=70)$ were approved by Jilin university ethical committee. Written consent forms were filled out prior to the blood sample collection. This study retrospectively enrolled patients who had previously undergone SCI between July 2010 and August 2012 that were identified from a search of the archival surgical pathology files of the China-Japan Union Hospital, Jilin University, Changchun, Jilin Province, China.Expert neurologist and neurosurgeon were involved in the validation of SCI based on the clinical findings (MRI and CT scan) and paraclinical manifestations. Age- and sex matched healthy control volunteers were recruited from among individuals who sought a routine health check-up at the Physical Health Examination Centre of Jilin University, who had not previously been diagnosed with SCI. Patients with no injury to CNS were recruited (age- and sex-matched to SCI cohort) to study the effect of NSCI on miR-21 levels. NSCI group $(n=60)$ included 34 arm injuries and 36 leg injuries. Serum preparation A $4 \mathrm{ml}$ sample of peripheral venous blood as drawn from all study participants after an overnight fast and placed at room temperature for $60 \mathrm{~min}$. Then the blood samples were centrifuged at $700 \mathrm{~g}$ for 10 min at $4{ }^{\circ} \mathrm{C}$ in a centrifuge, and then the serum was immediately separated from the 
blood, frozen and stored at $-80^{\circ} \mathrm{C}$ until used. During the sample storage, repeated freeze-thawing was avoided to ensure the quality of the samples. RNA extraction and quantitative RT-PCR analysis of miR-21 Total RNA was isolated using TRIzol (Invitrogen, CA) and miRNeasy mini kit (Qiagen, West Sussex, UK) according to manufacturer's instructions. This efficiently recovered all RNA species, including mi-RNAs. RNA quality and quantity was measured using a nanodrop spectrophotometer (ND-1000, Nanodrop Technologies) and RNA integrity was determined by gel electrophoresis.Total RNA from each sample was reverse-transcribed to cDNA using the PrimeScript RT reagent Kit (TaKaRa, Tokyo, Japan) and qRT-PCR was performed using the SYBR Premix Ex Taq (TaKaRa, Tokyo, Japan) and miRNA-specific primers for miR-21 (Ribobio, Guangzhou, China). The relative microRNA levels were normalized to U6 expression for each sample. The cycle threshold $(\mathrm{Ct})$, which was defined as the number of PCR cycles required for the fluorescent signal to be higher than a threshold indicating baseline variability, was recorded. Relative changes of gene expression were represented by $2-\Delta \Delta \mathrm{Ct}$, the difference between the original copy number of miR-21 in the NSCLC group and that in the control group. $\Delta \Delta \mathrm{Ct}=(\mathrm{Ct}$ miR-21-Ct U6) of the SCI group $-(\mathrm{Ct}$ miR-21-Ct U6) of the control group. Three repeated wells were set. Statistical analyses Statistical analyses were undertaken using GraphPad Prism version 5.01 (GraphPad Software, San Diego, CA, USA) and the SPSS ${ }^{\circ}$ statistical package, version 16.0 (SPSS Inc., Chicago, IL, USA) for Windows®. Statistical comparisons between data sets were made based on the representation of the mean \pm SD of data. Statistical analyses were performed using two-way repeated measurments analysis of variance (ANOVA) with Tukey HSC post hoc test for multiple comparisons. Significance level of p-value $<0.05$ was adopted.

\section{Results}

Data on the age of SCI patients and the cause and nature of the trauma are given in Table 1. Statistical analysis of demographic parameters included the mean age and gender. The mean age of the surviving patients was $35.12 \pm 3.46$ years. Of these, 36 patients age were above 35(older), the other were below 35(younger). Most of the patients $(71.11 \%)$ encountered SCI from automobile accidents and the rest from falling. 35.56\% of the patients had cervical SCI and the rest had SCI at all other levels of the spine. Of these, 48 patients were complete paralysis of spinal and 4248 patients were incomplete paralysis of spinal. We have recorded $8.89 \%$ mortality of SCI patients during the study period. NSCI patients were recruited to age- and sex-matched with the SCI cohort. NSCI group had mean age $39.44 \pm 4.75$ years (26 female and 34 male) and included 32 arm injuries and 28 leg injuries. The control group $(n=70)$ was composed of 40 males and 30 females (mean \pm SD age $37.3 \pm 3.4$ years). There was no significant difference in age and sex distribution between the patients with SCI and the control subjects. In the present study, we have quantitated the serum level of miR-21 of SCI patients at 1days, 7 and 28 days, and 3 months (Late) after the trauma. The data are given in Figure1. In the SCI patients, the serum level of miR-21 significantly increased 1day following the trauma. The increase in the serum level of miR-21 reached a peak on day 7 after SCI. After that period, the level of miR-21 returned back to the control level. However, there was no change in the serum miR-21 in NSCI patients and health control. There was no correlation between the level of miR-21 and patients gender (Figure2A). In addition, the expression the level of miR-21 has no correlation with patients age (Figure2B). There was a significant 
difference between the serum levels of miR-21 and different degree of injury ( $\mathrm{p}<$ $0.001)$. The serum level of miR-21 in complete paralysis of spinal group was significantly higher than those of incomplete paralysis of spinal group at 1day and 7day after SCI.

Table1. Demographics of SCI patients $(n=90)$ involved in the present study.

\begin{tabular}{|c|c|c|c|c|c|}
\hline \multirow{2}{*}{ Factor } & & \multicolumn{3}{|c|}{ Number of patients } & \multirow{2}{*}{ Age year (mean \pm SD) } \\
\cline { 2 - 5 } & & Male & Female & All & \\
\hline \multirow{2}{*}{ Cause of injury } & Vehicle & 46 & 18 & 64 & $36.12 \pm 6.78$ \\
\cline { 2 - 5 } & Falling & 16 & 10 & 26 & $34.34 \pm 5.12$ \\
\hline \multirow{2}{*}{ Site of injury } & Neck & 20 & 12 & 32 & $37.41 \pm 4.33$ \\
\cline { 2 - 5 } Outcome & Others & 42 & 16 & 58 & $35.20 \pm 5.56$ \\
\hline \multirow{2}{*}{ Degree of injury } & Death & 5 & 3 & 8 & $46.55 \pm 4.89$ \\
\cline { 2 - 5 } & Survived & 57 & 25 & 82 & $35.12 \pm 3.46$ \\
\cline { 2 - 5 } & $\begin{array}{c}\text { Complete paralysis } \\
\text { group }\end{array}$ & 30 & 18 & 48 & $37.14 \pm 6.73$ \\
\hline & $\begin{array}{c}\text { Incomplete paralysis } \\
\text { group }\end{array}$ & 32 & 10 & 42 & $35.22 \pm 4.98$ \\
\hline
\end{tabular}

A

B
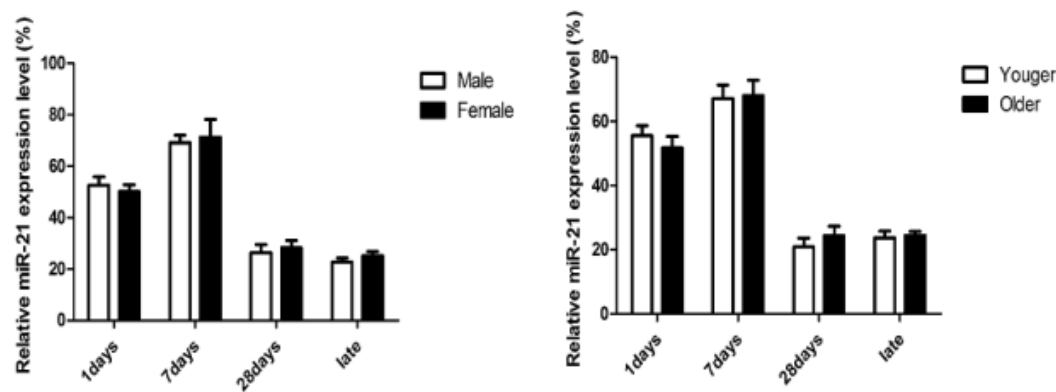

Figure2. Re lative level of micro RNA-21 (miR-21) in the serum after spinal cord injury (SCI) at different sex(A) and different age(B). Serum from SCI patients were collected 1, 7, 28 days and at three months ( $>30$ days) after a sustained SCI (late).

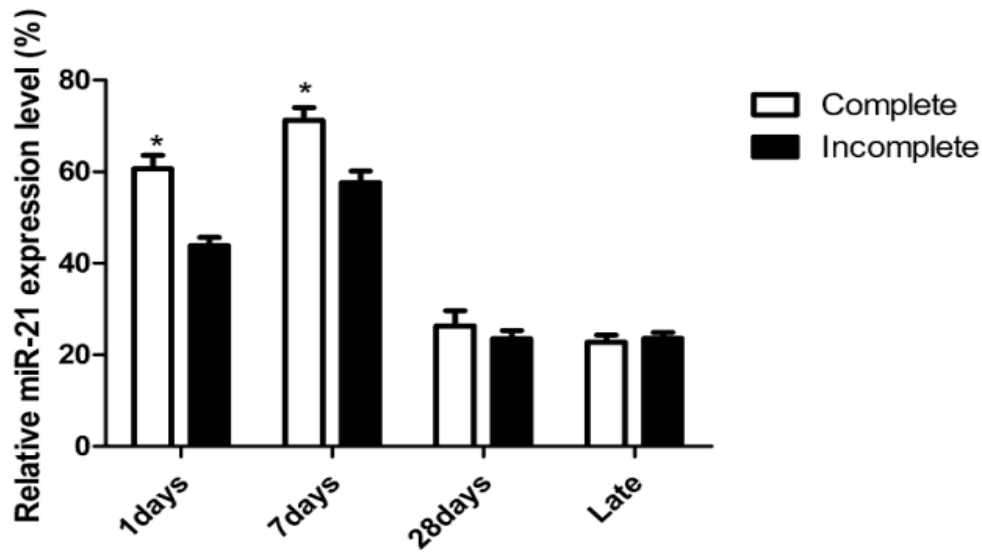

Figure3. Relative level of micro RNA-21 (miR-21) in the serum after spinal cord injury at complete paralys is of spinal group and incomplete paralysis of spinal group. ${ }^{*} \mathrm{P}<0.05$. 


\section{Discussion}

Traumatic spinal cord injury (SCI) could induces widespread molecular and biochemical changes, such as altered mRNA and protein expression,production of free radicals, axonal plasticity, inflammatory activation and neuronal cell death.18,19 Recently, it has been demonstrated that individual miRNAs can regulate hundreds of genes simultaneously by targeting RNA-induced silencing complexes (RISC) to mRNAs where they acted to inhibit translation or direct destructive cleavage. 20 Therefore, altering the expression of miRNAs may greatly affect SCI pathophysiology and functional outcome. In present study, we found that the serum levels of miR-21 at different time points has different changs after SCI, which further showed that miRNAs may greatly affect SCI pathophysiology and functional outcome. Recently, several other studies have demonstrated aberrant expression of miR-21 in many injury models. For example, microarray analyses demonstrated that the levels of many miRN As were altered at several time points (6-72 h) after traumatic brain injury, and that miR-21 was upregulated at all times in the rat cerebral cortex, 13 Similarly, Redell et al.found that the expressions of many miRNAs were altered in the hippocampus after traumatic brain injury, and miR-21 expression was significantly upregulated in the hippocampus with expression levels peaking 3 days post-injury, and returned to near normal levels days post-injury.21 In addition, recently, several studies demonstrated that miR-21 was upregulated following contusion SCI.10-12 Recently $\mathrm{Hu}$ et al reported miRNA signatures after SCI have revealed that multiple miRs are aberrantly expressed via microarray analyses. Among them, miR-21 expression upregulate and contributes to SCI. Knockdown of miR-21 in vivo exacerbates the functional deficit, aggravates tissue damage, and increases apoptotic cell death in rats following SCI.22 However, these report mainly focused on miR-21 expression in rat model, which have some different from patients. In the present, we selected patients as study objective and found the serum levels of miR-21 at different time points has different changs after SCI, which might contribute to the identification of selective and temporal drug targeted therapy after SCI. In conclusion, in the present study, our data showed that the serum levels of miR-21 at different time points has different changes after SCI and serum miR-21 levels has significantly correlated with degree of injury in SCI patients. These findings might contribute to the identification of selective and temporal drug targeted therapy after SCI.

Declaration of conflicting interest. The authors declare that there are no conflicts of interest.

Funding. The authors gratefully acknowledge the financial support provided by The Development of Science and Technology Plan Projects of Jilin (no. 2011Z099).

\section{Reference}

[1] Chaitanya GV, Kolli M, Babu PP. Granzyme-b mediated cell death in the spinal cord-injured rat model. Neuropathology 2009, 29: 270-279.

[2] Thuret S, Moon LD, Gage FH. Therapeutic interventions after spinal cord injury. Nat. Rev. Neurosci 2006; $7: 628-643$. 
[3] Carlson GD, Gorden C. Current developments in spinal cord injury research. Spine J 2002; 2:116-128.

[4] Dumont RJ Okonkwo DO, Verma S H. Acute spinal cord injury, part I: pathophysiologic mechanisms. Clin Neuropharmacol 2002 24:254-264.

[5] Bartel DP. MicroRNAs: genomics, biogenesis, mechanism, and function. Cell 2005; 116:281-297.

[6] Friedman RC, Farh KK, Burge CB, et al. Most mammalian mRNAs are conserved targets of microRNAs. Genome Res 2006; 19: 92-105.

[7] Kosik KS. The neuronal microRNA system. Nat Rev Neurosci 2006; 7: 911-920.

[8] Bak M, Silahtaroglu A, Møller M, et al. MicroRNA expression in the adult mouse central nervous system. RNA 2008;14:432-444.

[9] Johnson R, Zuccato C, Belyaev ND et al. A microRNA-based gene dysregulation pathway in Huntington's disease. Neurobiol Dis 2008; 29:438-445.

[10] Liu NK, Wang XF, Lu QB, et al. Altered microRNA expression following traumatic spinal cord injury. Exp Neurol 2009; 219: 424-429.

[11] Yunta M, Nieto-Di'az M, Esteban FJ, et al. MicroRNA dysregulation in the spinal cord following traumatic injury. PLoS One 2012; 7e34534.

[12] Strickland ER, Hook MA, Balaraman S, et al. MicroRNA dysregulation following spinal cord contusion: implications for neural plasticity and repair. Neuroscience 2011;186: 146-160.

[13] Lei P, Li Y, Chen X, et al. Microarray based analysis of microRNA expression in rat cerebral cortex after traumatic brain injury. Brain Res 2009; 1284: 191-201.

[14] Zhang L, Dong LY, Li YJ, et al. miR-21 represses FasL in microglia and protects against microglia-mediated neuronal cell death following hypoxia/ischemia. Gila 2012; 60: 1888-1895.

[15] Redell JB, Liu Y, Dash PK. Traumatic brain injury alters expression of hippocampal microRNAs: potential regulators of multiple pathophysiological processes. J Neurosci Res 2009; 87:1435-1448.

[16] Buller B, Liu X, Wang X. MicroRNA-21 protects neurons from ischemic death. FEBS J 2010; 277: 4299-4307.

[17] Dong S, Cheng Y, Yang J, et al. MicroRNA expression signature and the role of microRNA-21 in the early phase of acute myocardial infarction. J Biol Chem 2009, 29:514-525.

[18] De Biase A, Knoblach SM, Di Giovanni S, et al. Gene expression profiling of experimental traumatic spinal cord injury as a function of distance from impact site and injury severity. Physiol Genomics 2005; 22:368-381.

[19] Di Giovanni S, Knoblach SM, Brandoli C, et al. Gene profiling in spinal cord injury shows role of cell cycle in neuronal death. Ann Neurol 2003; 53:454-468.

[20] Baek D, Villen J, Shin C, et al. The impact of microRNAs on protein output. Nature 2008; 455: 64-71. 
[21] Redell JB, Zhao J, Dash PK. Altered expression of miRNA-21 and its targets in the hippocampus after traumatic brain injury. J Neurosci Res 2011; 89: 212-221.

[22] Hu JZ, Huang JH, Zeng L, et al.Anti-Apoptotic Effect of MicroRNA-21 after Contusion Spinal Cord Injury in Rats. J Neurotrauma.2013; 30:1349-1360 